\title{
Analysis on the Curative Effect of Minimally Invasive Percutaneous Renal Puncture Stone Extraction on the Patients with Urinary Calculi with Tuberculous
}

\author{
He $\operatorname{Tian}^{1, a}$, Yancheng $\mathrm{Di}^{1{ }^{1, *}, \text { Jixiang Bai }}{ }^{1}$ \\ ${ }^{1}$ Department of Urinary surgery, Hongqi Hospital, Mudanjiang Medical University, Aimin District, \\ Mudanjiang, China \\ ahetian@126.com
}

*Corresponding author yan888@163.com

Keywords: Quercetin; Ischemia reperfusion injury of the liver; Protect

\begin{abstract}
To investigate the urinary calculi with tuberculous patients treated with minimally invasive percutaneous renal puncture lithotomy curative effect, safety and its complications, thus it can provide the effective solution for the treatment of patients with urinary calculi with tuberculous. Methods: From June 2013 to August 2014 on our hospital 72 cases of urinary stones with tuberculous patients were randomly divided into observation group and the control group $(\mathrm{n}=36)$. The observation group 36 cases were treated by minimally invasive percutaneous renal puncture for the treatment of the lithotomy, while the control group is treated with the open operation. We observe two groups of the stones taking net rate, operation time, and the intraoperative blood loss, the hospital stay and the complications such as the index. Results: By the treatment and observation by two groups patients, the stones from net rate differences had no statistical significance between the observation group and control group $(\mathrm{P}>0.05)$; The operation time, blood loss, hospital stay of the observation group were significantly less than the control group $(\mathrm{P}<0.05)$; The observation group compared with preoperative creatinine postoperative $5 \mathrm{~d}$ has no obvious change. Conclusion: The minimally invasive percutaneous renal stone extraction for the treatment on urinary stones is safe, effect, and worth clinical promotion.
\end{abstract}

\section{Introduction}

With the continuous development of medical cause, clinical treatment gradually towards the direction of the minimally invasive surgery and transformation, this mainly lies in the minimally invasive surgery with small trauma, convenient, and the advantages of shorter operation time and party, was welcomed by the medical profession. For example, the application of minimally invasive surgery in urology in recent years is very much. In urology, there is a common clinical disease is on urinary stones, conventional treatment on urinary stones with bladder under ureteroscopy take path of urine road stone surgery treatment, this is a method for the treatment of urinary stones on the traditional, according to the practical experience and consult relevant references, the author learned this method operation one-time success rate is not high, only about $70 \%$. To this, there are many medical experts and scholars to explore an efficient, small trauma, platoon stone rate is high and the convenience of the operation treatment means and methods, which is minimally invasive percutaneous renal puncture nephrolithotomy (Mini - PNCL), the method is very simple to operate, and for patients with trauma is small, has won the praise of doctors and patients. Percutaneous nephroscope is intracavitary urology surgery in a very important part of ureteroscopy, extracorporeal shock wave lithotripsy and common as modern urinary calculi treatment. The application of these methods is gradually changing and replaced the traditional open surgery single treatment; make the kidney, the treatment is increasingly perfect. From June 2013 to August 2014 on our hospital 72 cases of urinary stones with tuberculous patients were randomly divided into observation group and the control group $(n=36), 36$ observation group underwent minimally invasive percutaneous renal puncture for the treatment of lithotomy, obvious curative effect, now report as follows. 


\section{The Materials and Methods}

\section{Materials}

We select our hospital 72 cases of urinary stones with tuberculous patients from June 2013 to August 2014 are randomly divided into observation group and control group 36 cases, observation group 36 cases (20 male and female in 16 cases, aged 25 to 67, average (46.8 99.4 ). Control group of 36 cases of male 21 cases, 15 cases were female, aged $23 \sim 65$, the average (47.2 \pm 10.1$)$ years of age. Two groups of patients are no significant differences in age, sex and disease, comparable.

\section{Methods}

The observation group of 36 cases was treated by minimally invasive percutaneous renal puncture for the treatment of lithotomy, preoperative preparation: (1) regular blood. Urine and biochemical examination and listen is to put the same operation. Preoperative the KUB, IVP, urine bacteria culture. (2) it is necessary for radionuclide renography, three-dimensional CT restructuring. (3) The surgery is introduced and knows that it makes patients prone position adaptability. The stones into the road choice of minimally invasive percutaneous renal biopsy technique using ureter mirror instead of kidney surgery, smaller channels and ureteroscopy in the elasticity of ureter mirror can make both greater scope for renal collection system, the minimally invasive percutaneous renal biopsy taken with the traditional percutaneous nephrolithotomy people road. Nephrolithotomy is different. Minimally invasive percutaneous renal puncture take nephrolithotomy in ureteral, pelvic stones, renal calyx calculi and merge UPJ obstruction that needs to be cut inside, at the same time into the road are the preferred light path, the puncture point often choose 11 after axillary line between ribs and the area between the shoulder line. While the control group treated with open operation. Both groups are to be $\mathrm{n} / \mathrm{med}$ tuberculosis medicine for treatment. Observing two groups of stones take net rate, operation time, intraoperative blood loss, hospital stay and complications such as index.

\section{Statistical processing}

With mathematical statistics software SPSS19.0 for data collection and statistical analysis, and chi-square test, $\mathrm{P}<0.05$, significant difference has statistical significance.

\section{Results}

\section{The lithotomy successful rate of two groups}

Through two groups of patients with treatment and observation, the observation group and control group the stones from net rate difference have no statistical significance $(P>0.05)$. As shown in table 1.

Table 1: Compared two groups of the lithotomy success rate

\begin{tabular}{ccccc}
\hline Group & $\mathrm{n}$ & $\begin{array}{c}\text { I stage } \\
\text { lithotomy }\end{array}$ & $\begin{array}{c}\text { II stage } \\
\text { lithotomy }\end{array}$ & $\begin{array}{c}\text { Taking the } \\
\text { net rate/\% }\end{array}$ \\
\hline The observation group & 36 & 34 & 2 & 100.0 \\
The control group & 36 & 32 & 3 & 97.22 \\
$\chi 2$ & & 0.086 & 0.871 & 0.128 \\
P value & & $P>0.05$ & $P>0.05$ & $P>0.05$ \\
\hline
\end{tabular}

\section{The surgery with two groups of patients}

The operation time, blood loss, hospital stay of the oobservation group are significantly less than the control group $(\mathrm{P}<0.05)$; Postoperative $5 \mathrm{~d}$ group compared with preoperative creatinine has no obvious change, the observation group of preoperative serum creatinine and blood urea nitrogen was $95.67 \pm 18.79 \mu \mathrm{mol} / \mathrm{L}, 9.32 \pm 1.32$ tendency for $\mathrm{L}$, postoperative serum creatinine and blood urea nitrogen was $94.26 \pm 17.62 \mu \mathrm{mol} / \mathrm{L}, 7.38 \pm 1.28$ tendency for $\mathrm{L}$, compared the two groups, 
serum creatinine level did not statistically significant, $\mathrm{P}>0.05$, blood urea nitrogen level significant differences statistically significant, $\mathrm{P}<0.05$ ).

Table 2: Two groups of operation time, blood loss and hospital stay

\begin{tabular}{|c|c|c|c|c|}
\hline Group & $\mathrm{n}$ & $\begin{array}{c}\text { The } \\
\text { operation } \\
\text { time (min) }\end{array}$ & $\begin{array}{l}\text { Blood loss } \\
\quad(\mathrm{ml})\end{array}$ & $\begin{array}{l}\text { The length of } \\
\text { time (d) }\end{array}$ \\
\hline The observation group & 36 & $\begin{array}{c}128.76 \pm \\
30.2\end{array}$ & $82.6 \pm 5.4$ & $5.1 \pm 1.8$ \\
\hline The control group & 36 & $178.4 \pm 42.8$ & $208.7 \pm 14.3$ & $8.5 \pm 1.8$ \\
\hline$\chi^{2}$ & & 12.763 & 14.197 & 8.645 \\
\hline $\mathrm{P}$ value & & $P<0.05$ & $P<0.05$ & $P<0.05$ \\
\hline
\end{tabular}

\section{Discussion}

Urinary calculus is a common disease of the urinary tract, stones seen in any part of the kidney, bladder, ureter and urinary tract. But with renal and ureter stone is for common. Clinical manifestations for stones is in different areas and different. Urinary tract tuberculosis mostly secondary to tuberculosis, cause renal tuberculosis disease mainly affects the kidney, spread to the bladder to the typical clinical symptoms: urinary frequency, urgency, urine or pyuria, may be accompanied by low grade fever, weight loss, fatigue, anemia and so on. Urinary tuberculosis (TB) is a kind of common disease, caused by mycobacterium tuberculosis blood line spread. The primary lesions in the lungs, tuberculosis, are about $1 \% \sim 4 \%$ with urinary tract tuberculosis. N/med tuberculosis bacterium menstrual cycle infringement of the kidney, forming nodules within the glomerulus's, if cannot heal, by again. Kidney spread to the ureter, and bladder. There are a few cases can be made of the spine, bowel and spread directly. Urinary tuberculosis (TB) is a systemic disease, we must attach importance to the whole body treatment, such as nutrition, environment, rest, the progress of the modern chemical treatment, in the principle of the treatment of urinary tract tuberculosis change, mainly drug treatment, with the necessary surgical treatment, early urinary tuberculosis (TB) becomes lighter, scope limits. After the proper use of anti-TB drugs treatment, can cure.

On urinary stones caused by the following factors, abnormal anatomy of the stone, such as urinary tract obstruction, narrow to the substrate or crystal deposition in poor drainage area, must urine retention cause urinary tract infections, create conditions for stone formation; Urine factors: have stone material to form in the urine, such as increased uric acid, calcium, higher $\mathrm{pH}$, reduced the volume of urine, higher organic matter and salt concentration, to a certain extent, reduces the inhibiting crystal form material content in the urine, such as acid gourd ladle polysaccharide, pyrophosphate, etc; High disease factors, such as idiopathic disease of urinary calcium, increase the quantity of the intestinal absorption of calcium, glomerular acidosis, caused by increased urine calcium excretion; Dietary factors: insufficient intake of vitamin B6, vitamin D is overmuch, easy to cause the urinary stones. According to patients' stone size, location, presence of urinary tract obstruction and infection, also exist differences in clinical manifestation. Pain is the most common symptoms, the persistent or paroxysmal, often ridge is located in the abdomen, rib Angle, can show the colic or dull pain, and to the vulva, lower abdomen radiation.

Percutaneous nephroscope technique (the pereutaneous nephroscopy) is through the establishment of from the skin to the renal collecting system operation channel, endoscopic enter calyces and renal pelvis, within the kidney disease diagnosis and treatment of a surgical method. Percutaneous nephroscope technology is cavity technology is an important part of urology, especially in the treatment of urinary stones, together with ureteroscopy technique and extracorporeal shock wave lithotripsy become modern main treatments, have completely changed the traditional way of surgical treatment of open surgery. In recent years, along with the clinical practice and experience accumulation, the improvement of technology and equipment, its operation 
method had the very big development, treatment scope expanding gradually. In recent years, along with the clinical practice and experience accumulation, the improvement of technology and equipment, its operation method had the very big development, treatment scope expanding gradually.

Percutaneous nephroscope surgery history began in the 1940 s, 1941 Rupol and Brown used endoscopic surgery of renal colostomy remove residual stones after operation; Goodwin 1955 first reported percutaneous renal colostomy surgery successfully remove the obstructed kidney effusion, this paper proposes the methods of percutaneous renal puncture colostomy. Sense and Johannson application of percutaneous renal biopsy 1976 kidney channel set up skin take stone set of success. Therefore, can be thought of percutaneous nephrolithotomy in percutaneous renal colostomy was developed on the basis of the emerging intracavitary urology surgery. Wickbam and Kollett (1981) named the technology "percutaneous nephrolithotomy". Since the 1990 s, with the progress of the application of optical fiber and processing technology, lumen device is able to improve. Various types of slender endoscope, ultrasonic lithotripsy and pneumatic ballistic lithotripsy and holmium laser lithotripsy cavity application of broken stone also helped improve the technology of percutaneous renal. The accumulation of clinical experience is making the treatment success rate, reduce complications, operation scope. In addition to the urinary stones on, open surgery residual calculi. Renal connected with ureteral stricture or atresia, upper tract surgery urinary leakage complex situation, can by percutaneous renal and cavity technology processing. Accordingly, from the simple to remove urinary tract obstructive percutaneous renal colostomy surgery (percutaneous nephrostomy, $\mathrm{PCN}$ ) to the comprehensive application in the urinary tract is given priority to with stones of the clinical application of many kinds of disease diagnosis and treatment technology, make the intracavitary urology got rapid development.

PCNL is the most important complication of intraoperative and postoperative bleeding, bleeding because of renal artery between segments or leaf damage. More than blind needle puncture, expansion tube into the kidney excess, excessive expansion channel cause renal laceration is a major cause of hemorrhage. Colostomy technology, the group with micro operation process under the TV monitor, is extremely low incidence of intraoperative and postoperative bleeding, no cases of open surgery hemostatic. The vast majority of intraoperative bleeding with kidney channel is along the thread to insert a larger fascia dilator tamponade oppression hemostasis, generally $20 \mathrm{~min}$ and stop bleeding; clip after eliminating blood clots may continue to operate. If bleeding again, should stop operation, insert the corresponding diameter of renal fistula, clip renal fistula oppression hemostasis. Postoperatively, rehydration, hemostatic drugs such as blood transfusion treatment when necessary.

On the clinical treatment of ureteral calculi, the ureter is larger, and for a long time, the long-term stimulation of ureteral mucosa, will form the kidney excessive effusion, form of inflammatory polyp parcel stone, distorts the ureter and kidney damage. The traditional treatment methods cannot effectively crushed stone; this application within the new operation work sheath can be inserted into the ureter, will thoroughly ejecting debris. In this study, patients with ureteral is on, platoon stone success rate as high as $100 \%$ under the effective treatment. Minimally invasive percutaneous renal puncture stone extraction for rib from 11 or 12 ribs between under the shoulder blade Angle line and axillary line under the area of puncture, at right angles to the spine, the operative field is open. If kidney seeper is not obvious, in order to improve the success rate of puncture, from ureter before puncture injection to form artificial kidney seeper, injection of contrast agent, observe morphological renal collecting system. Wear in the renal capsule, kidney seeper wear into the sense of a breakthrough, renal collecting system to pull out the needle core, urine flow, shows that the puncture success. Cut along the needle of the skin, fascia, then put the guide wire, plug to the ureter. If can't insert, at the same time to avoid fascia expansion godet sliding out, need to insert the thread more than $5 \mathrm{~cm}$. Then fascia dilator set in the thread, to promote equal depth, prevent twist thread or forward wear out to mucous membrane of the renal pelvis, requires the use of $\mathrm{C}$ arm fluoroscopy. If kidney stone is too big, in the $\mathrm{X}$ line for stone of puncture, injection of contrast agent, the manufacture of artificial kidney seeper, establish percutaneous channel, in placing 8.0/9.8 $\mathrm{F}$ ureter 
mirror, flushing cavity, keep clear operative field. The application of PEEL - away expanding sheath is near the stone, to flush platoon stone rubble. Application of micro create fistula operation, to avoid intraoperative bleeding, postoperative intraoperative hemorrhage along the thread to insert fascia dilator filling kidney channel oppression hemostasis, 20 min after blood check, in addition to blood clots to continue treatment. If the bleeding should be inserted into the renal fistula, clip made fistula, hemostasia after bleeding for the second phase of the treatment.

Using the ureteroscopy lithotomy, damage to the renal unit lighter, reducing the incidence of perioperative complications. Ureteroscopy can enter the calyces and because of the renal pelvis, avoid the percutaneous nephrolithotomy may cause calculi residual faults, make the completeness of gravel and stone effectively. This technology has the safety of ureteroscopy technique and nephroscope technology efficiency, has a high success rate of stone, for the most basic means of complexity in the treatment of kidney stones.

\section{Conclusion}

This data shows that the observation group and the control group stone taking net rate through two groups of patients with treatment and observation are no statistically significant difference comparing; the operation time, blood loss, and hospital stay of the observation group are significantly less than the control group. The treatment of the minimally invasive percutaneous renal stone extraction for urinary stones is safer, effect and worth clinical promotion.

\section{Acknowledgement}

This research was financially supported by the National Science Foundation.

\section{References}

[1] Hasan Serkan Dogan, Fuad Guliyev, Yesim S. Cetinkaya, Mustafa Sofikerim,Ender Ozden, Ahmet Sahin. Importance of microbiological evaluation in management of infectious complications following percutaneous nephrolithotomy[J]. International Urology and Nephrology. 2012(03)

[2] P. Tenke,B. Kovacs, M. Jäckel, E. Nagy. The role of biofilm infection in urology[J]. World Journal of Urology. 2012 (09)

[3] Iqbal Singh, Ashok Kumar, Praveen Kumar. "Ambulatory PCNL" (Tubeless PCNL under Regional Anesthesia) - A Preliminary Report of 10 Cases[J]. International Urology and Nephrology. $2012(09)$

[4] Rana A M, Bhojwani J P, Junejo N N,et al. Tubeless PCNL with patient in supine position: procedure for all seasons?--with comprehensivetechnique. Urology. 2012(10)

[5] Kunin M. Bridging septa of the perinephric space: anatomic, pathologic, and diagnostic considerations. Radiology. 2011(11)

[6] Chaussy C, Schmiedt E, Jocham D,et al. First clinical experience with extracorporeally induced destruction of kidney stones by shock waves. The Journal of Urology. 2012(08)

[7] Albala DM, Assimos DG, Clayman RV,et al. Lower pole I: A prospective randomized trial of extracorporeal shock wave lithotripsy and percutaneous nephrolithotomy for lower pole nephrolithiasis - initial results. The Journal of Urology. 2011(12)

[8] Evan AP, Willis LR, Mcateer $\mathrm{J}$ A,et al. Kidney damage and renal functional changes are minimized by waveform control that suppresses cavitation in shock wave lithotripsy. The Journal of Urology. 2012(07)

[9] Lahme S, Bichler KH, Strohmaier WL, et al. Minimally invasive PCNL in patients with renal pelvic and calyceal stones. European Urology. 2012(11)

[10] Sofer M, Watterson JD, Wollin TA,et al. Holmium: YAG laser lithotripsy for upper urinary tract calculi in 598 patients. Journal d'Urologie. 2012(06) 
[1] DavidW. Coldmana, Renato N. Pedryoc,Alex Kossettb,et al. Maximizing stone Fragmentation Efficiency With Ultrasonic Probes: Impact of Probe Pressure and Rotation. Journal d Urologie. 2012(02)

[12] Wickham JEA, Miller RA, Kellett MJ,et a1.Percutancous nephrolithotomy: one stage or two?. British Journal of Urology. 2012(06) 